\title{
A Flutter Prediction Framework in the Open-Source SU2 Suite
}

\author{
N. Simiriotis* and R. Palacios ${ }^{\dagger}$ \\ Imperial College London, London, SW7 2AZ, United Kingdom
}

\begin{abstract}
The paper reports on the development of a direct flutter-onset prediction framework, based on CFD frequency-domain techniques, for a robust and efficient search of the flutter boundary across the flight envelope. The implementation was carried out in the open-source SU2 solver. The complete methodology is examined in this contribution. The existing harmonic balance formulation was extended to treat arbitrarily deforming surfaces. A dedicated native solver was developed to integrate the linear structural equations of motion. Reduced order structural equations are built based on input modal shapes from external FE solvers. To this end, suitable interpolation schemes have been included to transfer data across the fluid-structure interface. This paper provides numerical results from the application of the developed approach to wellestablished 2D and 3D test cases operating in the transonic regime. The accuracy of the chosen strategy is shown, and the robustness and computational efficiency of the implementation are discussed.
\end{abstract}

\section{Introduction}

$\mathrm{C}^{\text {Lutter is a dynamic aeroelastic instability that causes self-sustained oscillatory vibrations. It is an undesirable }}$ F phenomenon for aircraft as it is detrimental to the aerodynamic performance and it can lead to comfort degradation of the ride, structural failure, or loss of control. Beyond the flutter point, nonlinearities in the structure (e.g., large deflections, follower loading) or in the aerodynamics (e.g., shock waves, flow separation) can cause finite-amplitude Limit Cycle Oscillations (LCOs). In order to, $(a)$ assure the robustness of the aircraft manufacturing, and $(b)$ shorten the development stage, it is important for the designers to have detailed and accurate information on the flutter sensitivity of the model and the aircraft behavior beyond the flutter point. Nowadays, this necessity is even more imperative since the industry increasingly searches for higher aspect-ratio solutions [1], and flexible aerodynamic surfaces to be used into morphing configurations [2].

The current standard for flutter prediction in the industry are techniques based on potential flow aerodynamics and CFD-based system identification methods. Linear potential-flow aerodynamic models, while very effective at subsonic flow conditions, are unable to predict the occurrence of shock waves, rendering inaccurate the prediction in transonic flow conditions. On the other hand, system identification sweeps over the design space and the flutter point is determined from the computed eigenvalues of the aeroelastic system. This approach can introduce transonic aerodynamics for each eigen-analysis through linear CFD-based Generalized Aerodynamic Forces (GAFs) computed in the frequency-domain. This, however, can be costly and non-robust due to system noise in the identification of the GAFs. Finally, high-fidelity CFD modeling and time-accurate coupled CFD-CSD calculations for large aircraft models are still prohibitively expensive to be used in a design framework.

Fortunately, in problems such as flutter where the periodic steady-state is of primary interest, frequency-based (or time-periodic) methods can be used to accelerate the solution process. Frequency-domain simulations present many advantages as they only seek the periodic solution directly, and bypass the initial transients in the unsteady problem. The basic idea of frequency-based methods is to represent all the state variables of the system with a Fourier series, and transform the time-dependent problem into a series of coupled steady state problems. In this way, the cost remains comparable to the steady problem solution. These highly efficient methods are applicable to systems of high complexity and can be used to track flutter instabilities across the flight envelope, and introduce naturally high-fidelity CFD simulations in the flutter prediction.

Frequency-domain flutter prediction is typically based on the harmonic balance (HB) method [3]. Variations of the classical balancing method include the nonlinear frequency domain (NLFD) form [4] and the time spectral (TS) form [5]. All methods assume that a periodic solution of the aeroelastic system exists for a given flight condition. Since flutter onset, however, is a self-excited vibration phenomenon, the corresponding frequency, and often the reduced velocity, are

\footnotetext{
*Research Associate, Department of Aeronautics, nikolaos.simiriotis@imperial.ac.uk.

†Professor of Computational Aeroelasticity, Department of Aeronautics, r.palacios@imperial.ac.uk, AIAA Associate Fellow.
} 
not known a priori. Therefore, specific strategies have been developed in order to search for the flutter velocity and response frequency simultaneously across the flight envelope.

Thomas et al. [6] first proposed a technique to determine the flutter and LCO response, based on unsteady transonic aerodynamics provided by the HB/CFD model. In their work, the HB method was used in conjunction with an inviscid CFD solver to determine the nonlinear aerodynamic force variation for the unsteady motions of a two-degree-of-freedom linear aeroelastic model. The flutter and LCO conditions were determined by prescribing finite values of pitching amplitude to the solver and performing Newton-Raphson iterations. Later, the same authors presented a modification to the so-called HB/LCO solver [7] that allowed for the aeroelastic governing equations to be solved simultaneously with the aerodynamic solver. In [7], the Newton-Raphson method was used every few iterations of the HB flow solver to determine all the aeroelastic variables, based on the aerodynamic loading at the current flow iteration. The authors demonstrated that the computational cost of the solution was independent of the number of structural degrees of freedom, and a well over an order of magnitude computational speed-up, compared to their original technique.

In a similar manner, He et al. [8] applied the TS method to create a high-fidelity flutter onset prediction methodology that could be used to capture the subcritical/supercritical LCO response (see Fig 1). The authors proposed a preconditioned, Jacobian-free, coupled Newton-Krylov solution strategy for the TS flutter equations, which resolved both the CSD and CFD equations in a robust and efficient manner. In their work, they assembled the dependent flow and structure variables into one global unknown vector which was then solved using a Newton-Krylov method. The use of Krylov iterative methods enforced fast solution for each linear equation encountered in Newton iterations. The Jacobian-free nature of the method further reduced the computational and memory cost of the method by eliminating the need to evaluate and store the full Jacobian of the aeroelastic system. The authors compared the proposed method against results acquired by time-marching (TM) simulations to demonstrate the accuracy and the efficiency of the approach.

Based also on the HB approach, Ekici et al. [9] developed an efficient "one-shot" method for determining self-excited LCOs in turbomachinery, and extended the method in [10] to predict LCOs for 2-DOF airfoils, in inviscid and viscous transonic flow regimes. In their work, the authors treated the LCO frequency as an unknown. The fluid and the structure fields were calculated by integrating the respective governing equations in pseudo-time, driving the residual of both fields to convergence simultaneously, while an optimization search was solving for the frequency, all in "one shot". Later, the authors modified this "one-shot" method [11] to capture the unstable LCO branch below the linear flutter point, and employed their approach to predict the onset of flutter [12]. In this improved approach, the amplitude of the deformation was prescribed as an input and the reduced velocity was treated, along with the response frequency, as unknown. The authors demonstrated that the proposed method was highly accurate in the problems treated, and showed that their approach offered significant computational savings compared to other prediction techniques.

The present paper examines the development of a direct flutter-onset prediction framework, based on the strategies that were covered in the introduction. The complete methodology will be examined in this contribution. The implementation of the developed framework was carried out in the open-source SU2 solver, in order to exploit the capabilities of a state-of-the-art CFD analysis package. SU2 is an open-source high-fidelity computational design tool [13] that contains a native fully coupled fluid-structure interaction solver and an HB implementation [14]. In this work, this HB implementation was further extended to treat arbitrarily deforming surfaces. The reduced order structural equations of motion are built based on input modal description of the configuration. This paper reports numerical results from the application of the developed approach to well-established 2D and 3D test cases operating in the transonic regime. The accuracy of the chosen strategy will be shown, and the robustness and computational efficiency of the implementation will be discussed. The work presented here has been funded by the COMAC-Imperial Research Centre for Wing Technology of Commercial Aircraft.

\section{Methodology}

\section{A. Fluid governing equation}

The Arbitrary Lagrangian-Eulerian (ALE) formulation of the Navier-Stokes equation, with grid displacement imposed at the structural boundary, is employed for the aeroelastic investigation. For time-dependent problems, the governing equation for compressible flow is given by:

$$
\frac{\partial \mathbf{w}}{\partial t}=-\mathbf{R}(\mathbf{w})
$$


with:

$$
\mathbf{R}(\mathbf{w})=\nabla \cdot\left(\mathbf{F}_{A L E}^{i n v}-\mathbf{F}^{v i s}\right)
$$

where $\mathbf{w}=\{\rho, \rho \mathbf{u}, \rho e\}^{T}$ are the conservative variables, and $\mathbf{F}_{A L E}^{i n v}, \mathbf{F}^{v i s}$ are the inviscid and viscous fluxes respectively, given by:

$$
\mathbf{F}_{A L E}^{i n v}=\left\{\begin{array}{c}
\rho(\mathbf{u}-\dot{\mathbf{z}}) \\
\rho \mathbf{u} \otimes(\mathbf{u}-\dot{\mathbf{z}})+\overline{\bar{I}} p \\
\rho e(\mathbf{u}-\dot{\mathbf{z}})+p \mathbf{u}
\end{array}\right\}, \mathbf{F}^{v i s}=\left\{\begin{array}{c}
0 \\
\overline{\bar{\tau}} \\
\overline{\bar{\tau}} \mathbf{u}+\mu C_{P} \nabla T
\end{array}\right\}
$$

The Euler equations can be derived by setting the viscous fluxes to zero. The pressure is given by the ideal gas law. The components of the viscous stress tensor $\overline{\bar{\tau}}$ are given by:

$$
\tau_{i j}=\mu\left(\frac{\partial u_{i}}{\partial x_{j}}+\frac{\partial u_{j}}{\partial x_{i}}-\frac{2}{3} \frac{\partial u_{k}}{\partial x_{k}} \delta_{i j}\right)
$$

The flow velocity vector $\mathbf{u}$ is written in the Cartesian system of reference, while $\dot{\mathbf{z}}$ is the local inertial velocity due to the mesh deformation. The mesh deformation is calculated from the boundary displacement of the structure by solving a pseudo-linear elastic problem defined as:

$$
K_{G} \mathbf{z}-f_{G}=0
$$

where $K_{G}$ is the fictitious stiffness matrix of the grid and $f_{G}$ is the vector of fictitious forces to enforce matching boundary displacements. This procedure satisfies the continuity of grid velocity across the interface.

\section{B. Harmonic Balancing}

Assuming that the flow is periodic and the structural vibrations are undamped, the dependent aeroelastic variables can be approximated as a truncated Fourier series for a prescribed number of harmonics $N_{H}$. A vector of conservative variables can be written for a specific fundamental frequency $\omega$ as:

$$
\mathbf{w}(\mathbf{x}, t)=\hat{\mathbf{w}}_{0}+\sum_{n=1}^{N_{H}}\left[\hat{\mathbf{w}}_{A, n}(\mathbf{x}) \cos (\omega n t)+\hat{\mathbf{w}}_{B, n}(\mathbf{x}) \sin (\omega n t)\right]
$$

where $\hat{\mathbf{w}}_{0}, \hat{\mathbf{w}}_{A, n}$ and $\hat{\mathbf{w}}_{B, n}$ are the spatial Fourrier coefficients of the variables. Eq. (6) can be rewritten in a discrete vector-matrix form as:

$$
\mathbf{w}^{*}=E^{-1} \hat{\mathbf{w}}
$$

where $\hat{\mathbf{w}}=\left\{\hat{\mathbf{w}}_{0}, \hat{\mathbf{w}}_{A, 1}, \hat{\mathbf{w}}_{B, 1}, \ldots, \hat{\mathbf{w}}_{A, N_{H}}, \hat{\mathbf{w}}_{B, N_{H}}\right\}^{T}$, and $\mathbf{w}^{*}=\left\{\mathbf{w}\left(t_{0}\right), \mathbf{w}\left(t_{1}\right), \ldots, \mathbf{w}\left(t_{N_{T}-1}\right)\right\}^{T}$ with $N_{T}=2 N_{H}+1$ and $t_{n}=2 n \pi / \omega N_{T}$. Conversely, if the solution is known in discrete time-instances, the Fourier coefficients can be determined from:

$$
\hat{\mathbf{w}}=E \mathbf{w}^{*}
$$

The vector of conservative variables and the unsteady residual in Eq. (1) can be approximated with a truncated Fourier series. Equating the sinus and cosinus parts (balancing) gives:

$$
\omega A \hat{\mathbf{w}}=-\hat{\mathbf{R}}
$$

where:

$$
A=\operatorname{Diag}\left(0, G_{1}, \ldots, G_{N_{H}}\right), \text { with } G_{n}=n\left[\begin{array}{cc}
0 & 1 \\
-1 & 0
\end{array}\right]
$$

Using Eq. (8), Eq. (9) can be cast back to the time domain:

$$
\begin{gathered}
\omega A E \mathbf{w}^{*}=-E \mathbf{R}^{*} \\
\omega \bar{D} \mathbf{w}^{*}=-\mathbf{R}^{*}
\end{gathered}
$$

where $\bar{D}=E^{-1} A E$. It can be seen that the HB approach results in $N_{T}$ steady systems, coupled through the pseudo-spectral operator $\omega \bar{D}$. Typically, to improve convergence, Eq. (11) is augmented with a pseudo time-marching term:

$$
\frac{\delta \mathbf{w}^{*}}{\delta \tau_{F}}+\omega \bar{D} \mathbf{w}^{*}=-\mathbf{R}^{*}
$$


The HB implementation in SU2 treats the $\omega \bar{D} \mathbf{w}^{*}$ part of the equation as a source term [14] and solves Eq. [12] iteratively. In this work, the existing SU2 implementation was modified in order to treat arbitrarily deforming surfaces in 3D problems. To this end, the volume variation in each time-instance should be taken into account, and Eq. 12] has to be augmented by the corresponding source terms:

$$
\frac{\delta\left(\mathcal{V} \mathbf{w}^{*}\right)}{\delta \tau_{F}}=-\tilde{\mathbf{R}}^{*}, \text { with } \tilde{\mathbf{R}}^{*}=\mathbf{R}^{*}+\omega \mathcal{V}^{*}\left(\bar{D} \mathbf{w}^{*}\right)+\omega \mathbf{w}^{*}\left(\bar{D} \mathcal{V}^{*}\right)
$$

where $\mathcal{V}^{*}=\left\{V\left(t_{0}\right), V\left(t_{1}\right), \ldots, V\left(t_{N_{T}-1}\right)\right\}^{T}$ is the vector containing the volume at each time-instance. The linear mesh solver is employed to compute the mesh deformation with respect to the original undeformed grid at each time-instance. Then, the grid velocity invoked in the ALE formulation, is approximated from the grid nodal coordinates at each instance as:

$$
\dot{\mathbf{z}}^{*} \approx \omega \bar{D} \mathbf{x}^{*}
$$

where $\dot{\mathbf{z}}^{*}=\left\{\dot{\mathbf{z}}\left(t_{0}\right), \dot{\mathbf{z}}\left(t_{1}\right), \ldots, \dot{\mathbf{z}}\left(t_{N_{T}-1}\right)\right\}^{T}$, and $\mathbf{x}^{*}=\left\{\mathbf{x}\left(t_{0}\right), \mathbf{x}\left(t_{1}\right), \ldots, \mathbf{x}\left(t_{N_{T}-1}\right)\right\}^{T}$

\section{Structural equation of motion}

The linear dynamic governing equations of motion under loading read:

$$
M \ddot{\boldsymbol{\eta}}+C \dot{\boldsymbol{\eta}}+K \boldsymbol{\eta}=\boldsymbol{f}
$$

where $M$ is the mass matrix, $C$ is the damping matrix, $K$ is the stiffness matrix, $\boldsymbol{\eta}$ is the displacement vector, and $\boldsymbol{f}$ the external loading derived from the aerodynamic forces. For flutter analysis, the first few structural modes are sufficient in providing an accurate prediction of the displacement. Typically, the number of retained mode shapes is much smaller than the structural degrees of freedom, reducing significantly the computational cost for large 3D structures. If the modal description of the structure is available (e.g., from a FE solver), it is possible to write Eq. (15) as:

$$
\begin{gathered}
\Phi^{T} M \Phi \ddot{\boldsymbol{q}}+\Phi^{T} C \Phi \dot{\boldsymbol{q}}+\Phi^{T} K \Phi \boldsymbol{q}=\Phi^{T} \boldsymbol{f} \\
M_{\phi} \ddot{\boldsymbol{q}}+C_{\phi} \dot{\boldsymbol{q}}+K_{\phi} \boldsymbol{q}=\boldsymbol{f}_{\phi} \\
M_{\phi} \ddot{\boldsymbol{q}}+M_{\phi} T_{\phi} \Omega \dot{\boldsymbol{q}}+M_{\phi} \Omega^{2} \boldsymbol{q}=\boldsymbol{f}_{\phi}
\end{gathered}
$$

where $\Phi=\left[\boldsymbol{\phi}_{1}, \boldsymbol{\phi}_{2}, \ldots, \boldsymbol{\phi}_{r}\right]$ are the first $r$ modal shapes, $\boldsymbol{q}=\Phi^{-1} \boldsymbol{\eta}$ are the generalized displacements, and $\boldsymbol{f}_{\phi}$ are the generalized forces. The matrix $M_{\phi}=\operatorname{Diag}\left(m_{1}, m_{2}, \ldots, m_{r}\right)$ contains the modal masses, while $T_{\phi}$ contains the modal damping factors, and can be assumed $T_{\phi}=\operatorname{Diag}\left(2 \xi_{1}, 2 \xi_{2}, \ldots, 2 \xi_{r}\right)$ if certain conditions apply for $C$. Finally, $\Omega=\operatorname{Diag}\left(\omega_{1}, \omega_{2}, \ldots, \omega_{r}\right)$ is the matrix containing the natural frequencies for the first $r$ natural modes retained. Similar to the fluid equations, the pseudo-spectral operator $\omega \bar{D}$ can be employed in order to acquire the HB set of equations:

$$
\begin{gathered}
\omega^{2} M_{\phi} \bar{D}^{2} \boldsymbol{q}^{*}+\omega M_{\phi} T_{\phi} \Omega \bar{D} \boldsymbol{q}^{*}+M_{\phi} \Omega^{2} \boldsymbol{q}^{*}=\boldsymbol{f}_{\phi}^{*} \\
\omega^{2} \bar{D}^{2} \boldsymbol{q}^{*}+\omega T_{\phi} \Omega \bar{D} \boldsymbol{q}^{*}+\Omega^{2} \boldsymbol{q}^{*}=M_{\phi}^{-1} \boldsymbol{f}_{\phi}^{*}
\end{gathered}
$$

where $q^{*}$ and $f_{\phi}^{*}$ contain the generalized displacements and loads, respectively, at discrete time-instances. The nondimensional form of Eq. (17) can be derived by normalizing the aerodynamic forces with the freestream dynamic pressure $q_{\infty}=\rho_{\infty} U_{\infty}^{2} / 2$, and introducing a reference mass $m_{0}$ for the wing:

$$
\tilde{\boldsymbol{f}}^{*}=\frac{m_{0} \boldsymbol{M}_{\phi}^{-1} \boldsymbol{f}_{\phi}^{*}}{q_{\infty} S}
$$

where $S$ is a reference surface. A reference length $b$ and a natural frequency (e.g., the first mode $\omega_{1}$ ) can be also introduced. Then, the dimensionless form of Eq. (17) reads:

$$
\left(\frac{\omega}{\omega_{1}}\right)^{2} \bar{D}^{2} \frac{\boldsymbol{q}^{*}}{b}+\frac{\omega}{\omega_{1}} T_{\phi} \frac{\Omega}{\omega_{1}} \bar{D} \frac{\boldsymbol{q}^{*}}{b}+\frac{\Omega^{2}}{\omega_{1}^{2}} \frac{\boldsymbol{q}^{*}}{b}=\frac{\rho_{\infty} U_{\infty}^{2} S}{2 m_{0} \omega_{1}^{2} b} \tilde{\boldsymbol{f}}^{*}
$$




$$
\tilde{\omega}^{2} \bar{D}^{2} \tilde{\boldsymbol{q}}^{*}+\tilde{\omega} T_{\phi} \tilde{\Omega} \bar{D} \tilde{\boldsymbol{q}}^{*}+\tilde{\Omega}^{2} \tilde{\boldsymbol{q}}^{*}=\frac{U_{\infty}^{2} S}{2 \mathcal{V} \mu \omega_{1}^{2} b} \tilde{\boldsymbol{f}}^{*}
$$

where $\tilde{\omega}$ is the nondimensional frequency, $\mu=m_{0} /\left(\rho_{\infty} \mathcal{V}\right)$ is the mass ratio, and $\mathcal{V}$ is the volume of the wing. By substituting the flutter index speed $\tilde{V}_{F}=U_{\infty} /\left(\sqrt{\mu} b \omega_{1}\right)$, Eq. (19) can be written:

$$
\tilde{\omega}^{2} \bar{D}^{2} \tilde{\boldsymbol{q}}^{*}+\tilde{\omega} T_{\phi} \tilde{\Omega} \bar{D} \tilde{\boldsymbol{q}}^{*}+\tilde{\Omega}^{2} \tilde{\boldsymbol{q}}^{*}=\tilde{V}_{F}^{2} \frac{S b}{2 \mathcal{V}} \tilde{\boldsymbol{f}}
$$

which constitute the nondimensional HB governing equations of generalized motion under aerodynamic loading. For this work, a native HB solver for the solution of Eq. 20] has been developed within the SU2 solver. In order to improve convergence, Eq. 20) can also be augmented with a pseudo time-marching term:

$$
\frac{\delta \tilde{\boldsymbol{q}}}{\delta \tau_{S}}+\tilde{\omega}^{2} \bar{D}^{2} \tilde{\boldsymbol{q}}^{*}+\tilde{\omega} T_{\phi} \tilde{\Omega} \bar{D} \tilde{\boldsymbol{q}}^{*}+\tilde{\Omega}^{2} \tilde{\boldsymbol{q}}^{*}-\tilde{V}_{F}^{2} \frac{S b}{2 \mathcal{V}} \tilde{\boldsymbol{f}}=0
$$

In general, the fluid $\delta \tau_{F}$ and structural $\delta \tau_{S}$ pseudo time-steps do not have to coincide. To simplify the notation, the will be dropped from here on.

\section{Grid interpolation method}

The modal description of the structure is expected to be given on an arbitrary structural mesh from an external FE solver. In the general case, the discrete interface between the fluid and the structural domain may be non-conforming, and the exchange of data becomes non-trivial [15]. To this end, an interpolation scheme is employed in order to transfer both displacements and loads across the fluid-structure interface. The coupling scheme must satisfy the conservation of energy across the interface [16, 17] which can be ensured by the equivalence of virtual work performed by the aerodynamic loads $\left(\delta W_{f}\right)$ and the structural forces $\left(\delta W_{s}\right)$ :

$$
\delta W_{s}=\delta \mathbf{u}_{s}^{T} \mathbf{f}_{s}=\delta \mathbf{u}_{f}^{T} \mathbf{f}_{f}=\delta W_{f}
$$

where $\delta \mathbf{u}_{*}$ is the virtual displacement, and $\mathbf{f}_{*}$ is the force vector. The deformation of the aerodynamic mesh can be expressed as a linear approximation of the structural deformation by introducing the coupling matrix $\bar{H}$ :

$$
\mathbf{u}_{f}=\bar{H} \mathbf{u}_{s}
$$

From the conservation of energy, i.e. Eq. 22], it can then be deduced:

$$
\mathbf{f}_{s}=\bar{H}^{T} \mathbf{f}_{f}
$$

In this work, a multivariate interpolation technique based on Radial Basis Functions (RBF) is implemented in the developed flutter prediction framework. The displacement vector $\vec{u}_{s}\left(\vec{x}_{n}^{s}\right)=\vec{u}_{s, n}=\left[\Delta x_{n}^{s}, \Delta y_{n}^{s}, \Delta z_{n}^{s}\right]^{T}$ for each structural grid node $\vec{x}_{n}^{s}=\left[x_{n}^{s}, y_{n}^{s}, z_{n}^{s}\right]^{T}$ can be expressed [18] as:

$$
\vec{u}_{s, n}=\sum_{i=1}^{N_{s}} \vec{\alpha}_{i} \psi\left(\left\|\vec{x}_{n}-\vec{x}_{i}\right\|\right)+\boldsymbol{p}\left(\vec{x}_{n}\right), \text { with } \vec{\alpha}_{i}=\left[\alpha_{i}^{1}, \alpha_{i}^{2}, \alpha_{i}^{3}\right]^{T}
$$

where $N_{s}$ is the number of structural grid nodes, $\psi$ are basis functions of the Euclidean distance, and $p\left(\vec{x}_{n}\right)$ is a polynomial. If basis functions are chosen to be conditionally positive definite functions of order $m \leq 2$, a linear polynomial $p$ can be used:

$$
\boldsymbol{p}\left(\vec{x}_{n}\right)=\left\{\begin{array}{l}
p_{1}\left(\vec{x}_{n}\right) \\
p_{2}\left(\vec{x}_{n}\right) \\
p_{3}\left(\vec{x}_{n}\right)
\end{array}\right\}=\bar{B}\left[\begin{array}{c}
1 \\
\vec{x}_{n}
\end{array}\right], \text { where } \bar{B}=\left[\boldsymbol{\beta}_{1}, \boldsymbol{\beta}_{2}, \boldsymbol{\beta}_{3}\right]^{T}=\left[\begin{array}{llll}
\beta_{1,0} & \beta_{1,1} & \beta_{1,2} & \beta_{1,3} \\
\beta_{2,0} & \beta_{2,1} & \beta_{2,2} & \beta_{2,3} \\
\beta_{3,0} & \beta_{3,1} & \beta_{3,2} & \beta_{3,3}
\end{array}\right]
$$

An additional requirement can be set for each dimension $j=1,2,3$ :

$$
\sum_{i=1}^{N_{S}} \alpha_{i}^{j} q_{j}\left(\vec{x}_{i}\right)=0
$$


where $q$ is any polynomial with a degree less than or equal to that of $p$. Without loss of generality one can set $q\left(\vec{x}_{n}\right)=1+x_{n}+y_{n}+z_{n}$ in every dimension. Eqs. 25] and (27) can be written together in a discrete vector-matrix form for each dimension (here $\mathrm{x}$-dimension):

$$
\left[\begin{array}{c}
\Delta \mathbf{x}^{s} \\
\mathbf{0}
\end{array}\right]=\left[\begin{array}{cc}
\bar{C}^{s s} & \bar{Q}_{s} \\
\bar{Q}_{s}^{T} & \overline{0}
\end{array}\right]\left[\begin{array}{c}
\boldsymbol{\alpha}^{1} \\
\boldsymbol{\beta}_{1}
\end{array}\right]
$$

where $\bar{C}_{n i}^{s s}=\psi\left(\left\|\vec{x}_{n}^{s}-\vec{x}_{i}^{s}\right\|\right)$, with $n, i=1, \ldots, N_{s}$, and $\bar{Q}_{s}=\left[\mathbf{1}, \mathbf{x}_{s}, \mathbf{y}_{s}, \mathbf{z}_{s}\right]_{N_{S} \times 4}$. Similar expressions can be deduced in the other dimensions. Eq. (28) can be used to calculate the $\boldsymbol{\alpha}$ and $\boldsymbol{\beta}$ coefficients from the structural displacement. Then, the displacements for the $N_{f}$ points of the fluid surface can be deduced from:

$$
\Delta \mathbf{x}^{f}=\left[\begin{array}{ll}
\bar{C}^{f s} & \bar{Q}_{f}
\end{array}\right]\left[\begin{array}{l}
\boldsymbol{\alpha}^{1} \\
\boldsymbol{\beta}_{1}
\end{array}\right]=\left[\begin{array}{ll}
\bar{C}^{f s} & \bar{Q}_{f}
\end{array}\right]\left[\begin{array}{cc}
\bar{C}^{s s} & \bar{Q}_{s} \\
\bar{Q}_{s}^{T} & \overline{0}
\end{array}\right]^{-1}\left[\begin{array}{c}
\Delta \mathbf{x}^{s} \\
\mathbf{0}
\end{array}\right]=\bar{H}\left[\begin{array}{c}
\Delta \mathbf{x}^{s} \\
\mathbf{0}
\end{array}\right]
$$

where $\bar{C}_{m i}^{f s}=\psi\left(\left\|\vec{x}_{m}^{f}-\vec{x}_{i}^{s}\right\|\right)$, with $m=1, \ldots, N_{f}$, and $m=1, \ldots, N_{s}$, and $\bar{Q}_{f}=\left[\mathbf{1}, \mathbf{x}_{f}, \mathbf{y}_{f}, \mathbf{z}_{f}\right]_{N_{f} \times 4}$. Various basis functions have been evaluated for fluid-structure interaction computations [15, 17]. In this work, two commonly used functions have been implemented and evaluated: $(i)$ the Thin Plate Spline (TPS) with global support,

$$
\psi(\|.\|)=\|\cdot\|^{2} \log (\|\cdot\|)
$$

and (ii) the Euclid's Hat (EH) with radial compact support of size $d$,

$$
\psi(\|.\|)=\pi\left(\frac{\|.\|^{3}}{12}-d^{2}\|.\|+\frac{4 d^{3}}{3}\right)
$$

Both approaches were successfully validated, and the EH was found to provide the best results for 3D configurations.

\section{E. HB/Flutter prediction}

The flutter prediction framework developed in SU2 is based on the work of Li \& Ekici [11, 12]. The coupled set of Eqs. (12) and (21) constitute the aeroelastic system in HB form. When searching for a flutter condition, both the frequency $\omega$ and the flutter velocity $V_{F}$ are unknown a priori. Thus, additional constraints should be added to the aeroelastic solution process in order to treat $\omega$ and $V_{F}$ as independent variables of the problem. One of the structural degrees of freedom (e.g., mode $q_{1}$ here) can be chosen arbitrarily to be constrained. Both the amplitude and the phase of the chosen mode must be fixed: $(a)$ the phase will provide an initial time reference for the periodic aeroelastic solution, and $(b)$ the chosen amplitude will uniquely fix the solution along the bifurcation diagram of the system (see Fig 1 . To drive the system towards the flutter boundary, it is sufficient to constrain the vibration amplitude to a small value $A_{o}$.

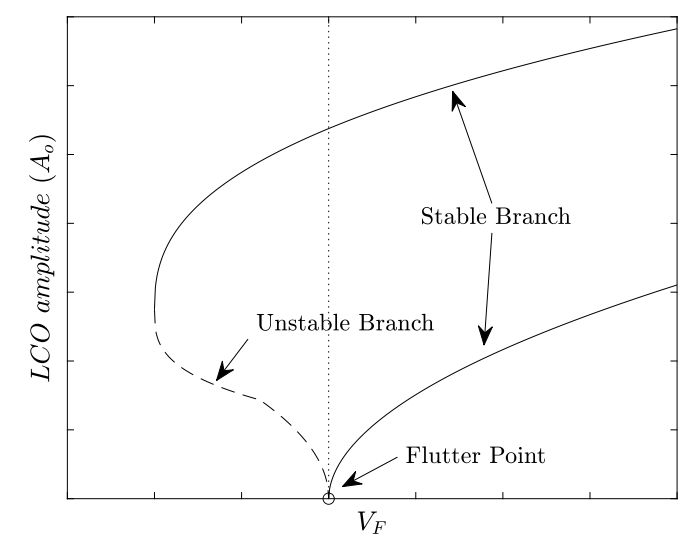

Fig. 1 Supercritical (i.e., above flutter point) and subcritical (i.e., below flutter point) bifurcation diagrams.

Since flutter is a linear instability, only one harmonic is sufficient when expanding each structural degree of freedom:

$$
q_{1}(t)=\hat{q}_{1,0}+\hat{q}_{1, A 1} \cos (\omega t)+\hat{q}_{1, B 1} \sin (\omega t)
$$


where $\hat{q}_{1,0}$ corresponds to the mean value of the mode, while the modal amplitude $A_{1}$ is defined as:

$$
A_{1}=\sqrt{\hat{q}_{1, A 1}^{2}+\hat{q}_{1, B 1}^{2}}
$$

The predicted phase of the mode is given from:

$$
\theta_{1}=\arctan 2\left(\hat{q}_{1, A 1} / \hat{q}_{1, B 1}\right)-\pi / 2
$$

Without loss of generality, the phase of the mode can be fixed by setting $\hat{q}_{1, A 1}=0$, while the amplitude is constrained by setting $\hat{q}_{1, B 1}=A_{o}$. Then,

$$
\mathbf{q}_{1}=\left[q_{1}\left(t_{0}\right), q_{1}\left(t_{1}\right), q_{1}\left(t_{2}\right)\right]^{T}=E^{-1}\left[\hat{q}_{1,0}, \hat{q}_{1, A 1}, \hat{q}_{1, B 1}\right]^{T}
$$

where $t_{i}=2 \pi i / 3 \omega$ for one harmonic. The rest of the structural modes should be shifted in accordance to retain the relative phase difference:

$$
q_{n}(t)=\hat{q}_{n, 0}+\hat{q}_{n, A 1} \cos \left(\omega t+\theta_{p}\right)+\hat{q}_{n, B 1} \sin \left(\omega t+\theta_{p}\right), \text { for every } n>1
$$

To relax the procedure, one could instead scale the modal amplitude with $\hat{q}_{1, A 1}^{\text {new }}=c \hat{q}_{1, A 1}^{\text {old }}$ and $\hat{q}_{1, B 1}^{\text {new }}=c \hat{q}_{1, B 1}^{\text {old }}$, where $c=A_{1} / A_{o}$. In this case, the chosen mode $q_{1}$ should then be shifted along with the rest of the modes using Eq. 36.

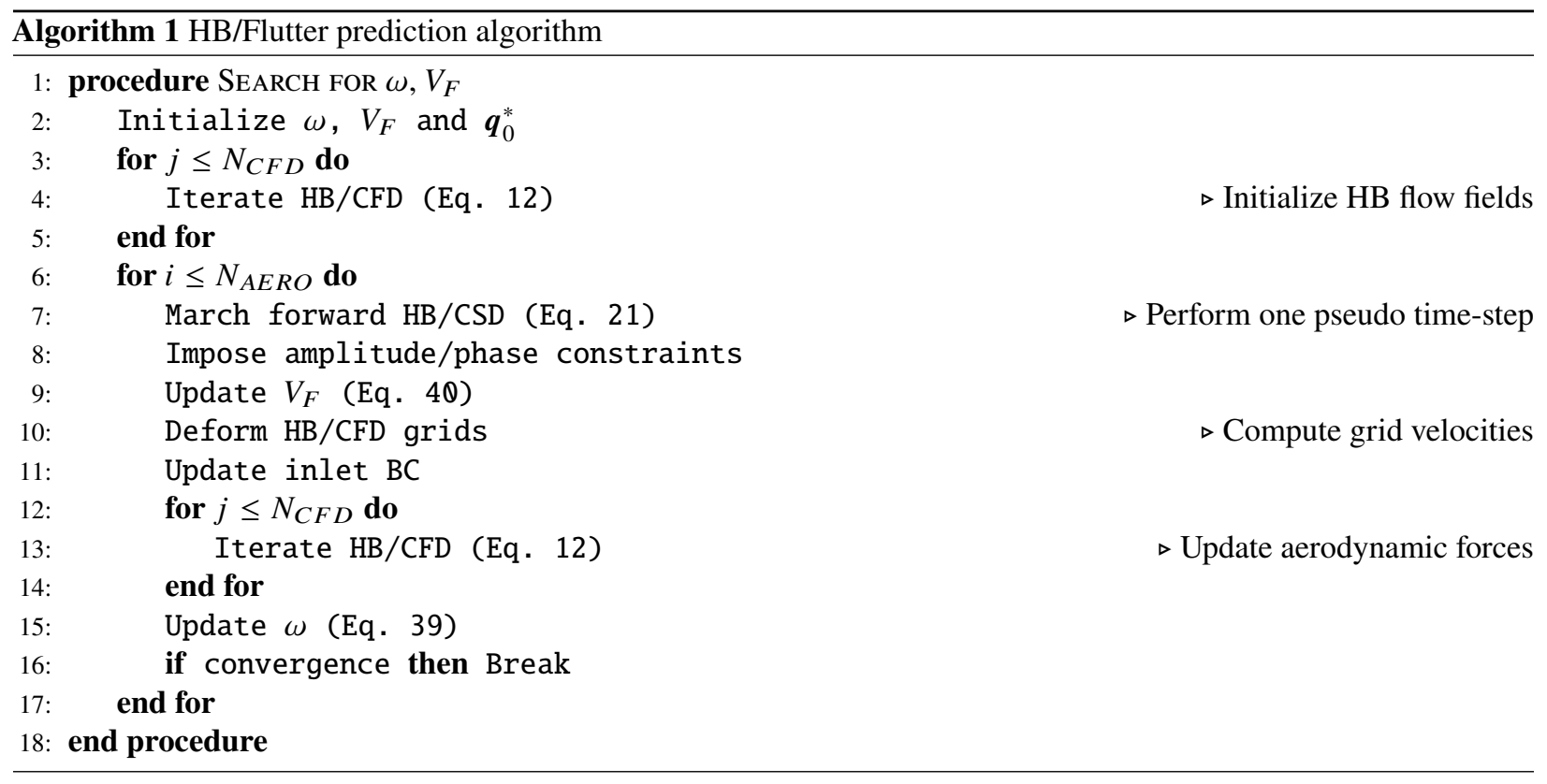

The above constraints allow for the flutter velocity and the frequency to be treated as independent variables. Their values should be updated at every aeroleastic iteration for the complete system to converge to a unique solution. This can be achieved by minimizing a figure of merit [19], based here on the structural residual [11]:

$$
\mathcal{L}\left(\omega, V_{F}\right)=\frac{1}{2} \boldsymbol{R}_{s}^{T} \boldsymbol{R}_{S}
$$

with:

$$
\boldsymbol{R}_{s}=\omega^{2} \bar{D}^{2} \boldsymbol{q}^{*}+\omega T_{\phi} \Omega \bar{D} \boldsymbol{q}^{*}+\Omega^{2} \boldsymbol{q}^{*}-V_{F}^{2} \frac{S b}{2 \mathcal{V}} \boldsymbol{f}
$$

The flutter frequency can be updated from:

$$
\frac{\partial \mathcal{L}}{\partial \omega}=\left(\frac{\partial \boldsymbol{R}_{S}}{\partial \omega}\right)^{T} \boldsymbol{R}_{s} \approx\left(\boldsymbol{q}^{*}\right)^{T}\left(2 \omega \bar{D}^{2}+T_{\phi} \Omega \bar{D}\right)^{T} \boldsymbol{R}_{s}=0
$$


where the flutter velocity is fixed at its previous value. Then, if the frequency is fixed, the flutter velocity can be updated in the same manner:

$$
\frac{\partial \mathcal{L}}{\partial V_{F}}=\left(\frac{\partial \boldsymbol{R}_{s}}{\partial V_{F}}\right)^{T} \boldsymbol{R}_{s} \approx 2 V_{F} \frac{S b}{2 \mathcal{V}} \boldsymbol{f}^{T} \boldsymbol{R}_{s}=0
$$

In both Eq. (39) and Eq. (40), the derivatives of the aerodynamic forces with respect to $\omega$ and $V_{F}$ respectively, are neglected. This approximation has been shown to not affect the prediction of the flutter solution [10], while it accelerates the solution process since the sensitivity of the forces significantly increases the computational cost.

Based on the previous discussion, a flutter/LCO prediction strategy has been developed and implemented in SU2. The procedure is shown in Algorithm 1. The solution is initialized and the aeroelastic system of HB equations is solved iteratively. At every aeroelastic iteration $N_{A E R O}, \omega$ and $V_{F}$ are updated in a decoupled manner, which enhanced the robustness of the searching strategy. The decoupling also allowed for fewer inner $N_{C F D}$ iterations to be used, thus, decreasing the computational cost. In this implementation, the velocity update is carried out after the CSD problem is solved and the amplitude/phase constraints are imposed. Based on the updated flutter velocity, the inlet boundary conditions (BC) for the flow are updated. Then, the CFD grids are computed for each time-instance with respect to the initial undeformed geometry. After the update of the aerodynamic forces, the new frequency is calculated. Finally, the updated aerodynamic forces and the new frequency are used to march forward the CSD problem in the next aeroelastic iteration. The whole procedure is repeated until $\omega$ and $V_{F}$ converge, and both the structural and the fluid residuals sufficiently decrease.

\section{Results}

In this part of the paper, the developed flutter prediction strategy is evaluated through numerical examples. First, the HB implementation in SU2 is validated for a 2D airfoil configuration and imposed kinematics. The accuracy of the unsteady force prediction is evaluated. Then, the HB/Flutter algorithm is used for the same configuration to construct its bifurcation diagram. The results are examined against the available data in the literature. Finally, a typical 3D configuration is examined for variable Mach number in order to highlight the accuracy and the robustness of the implementation in predicting the flutter boundary of wings.

\section{A. Imposed deformation}

As previously mentioned, the existing HB formulation in SU2 has been extended here in order to accommodate arbitrarily deforming surfaces. This allows to consider non-uniform surface displacements, as in the case of 3D deforming structures. The implementation has been tested for the imposed sinusoidal pitching motion of the NACA 64A010 airfoil, around a zero mean angle of attack, $\alpha_{0}=0(\mathrm{deg})$. The specifications of this test case can be found in Table 1 .

Table 1 Specifications for the imposed pitch test case.

\begin{tabular}{cccc}
\hline Pitching Amplitude & Mach Number & Reduced Frequency & Pitching axis \\
\hline $\bar{\alpha}=1.02(\mathrm{deg})$ & $M=0.769$ & $\tilde{\omega}=0.202$ & $e=0.248 c$ \\
\hline
\end{tabular}

The simulation has been carried out with the Euler solver of SU2 for $N_{H}=2$ harmonics, i.e., $N_{T}=5$ timeinstances. The Jameson-Schmidt-Turkel (JST) central scheme with scalar dissipation (second and fourth order dissipation coefficients) was employed for this study. The 2D computational O-type grid includes approximately 17000 triangular volume elements, with 200 volume elements placed around the boundary of the airfoil. The resolution of the mesh is satisfactory for inviscid computations. Freestream boundary conditions were set at the edge of the computational domain, and Euler (slip) walls on the aerodynamic surface.

The results are shown in Fig. 2 where the lift and the moment coefficient are plotted against the variation of the pitch angle. The reference point for the moment calculation is set at $0.25 c$, where $c$ is the aerodynamic chord of the airfoil. It can be seen that the SU2 results compare well with [10] even with only two harmonics, since the amplitude is kept at moderate levels. Both the amplitude and the relative phase difference between the two modes of vibration is accurately captured. The elliptical shape of the force variation highlights the aerodynamic effects of hysteresis that are also accurately captured. Finally, the HB results are also in agreement with time-accurate simulations that have been carried out with SU2, and not shown here in the sake of brevity. 


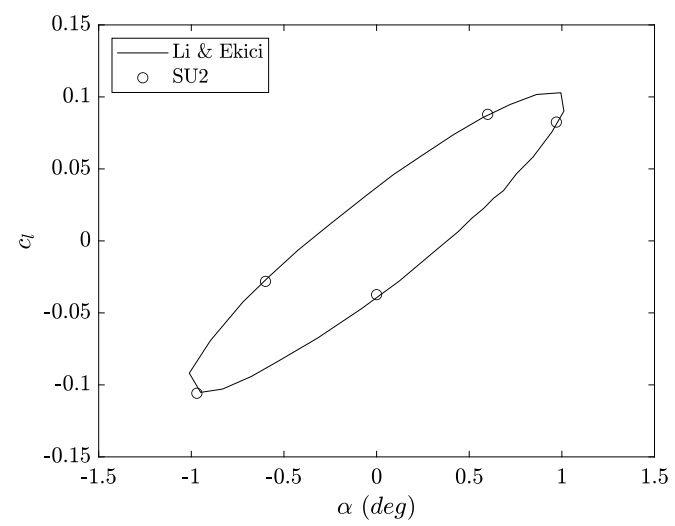

(a)

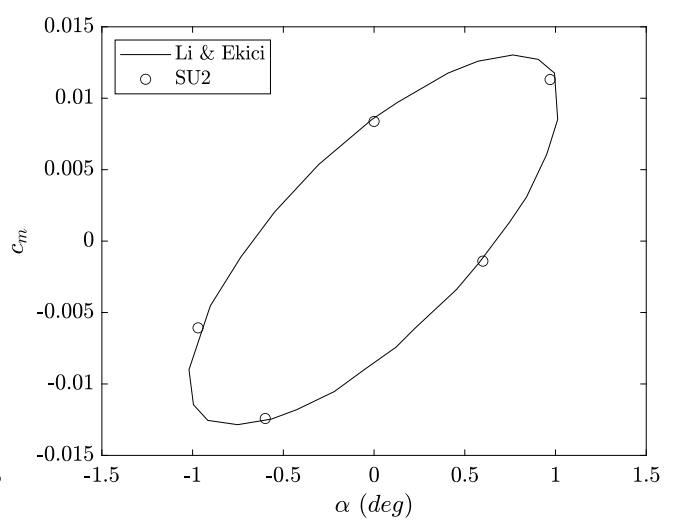

(b)

Fig. 2 Variation of (a) the lift, and (b) the moment coefficient during the pitching motion, plotted against the results found in Ref. [10]

\section{B. Flutter/LCO for plunge/pitch airfoil}

The flutter/LCO prediction strategy described in Section II.E is demonstrated here for a two-degrees-of-freedom aerolastic model. The governing aeroelastic equations, without damping, for an airfoil with plunge and pitch degrees-of-freedom, in nondimensional form [6], is:

$$
M \ddot{\boldsymbol{q}}+K \boldsymbol{q}=\frac{V_{F}^{2}}{\pi} \boldsymbol{f}
$$

with:

$$
\boldsymbol{q}=\left\{\begin{array}{l}
\frac{h}{b} \\
\alpha
\end{array}\right\}, M=\left[\begin{array}{cc}
1 & x_{\alpha} \\
x_{\alpha} & r_{\alpha}^{2}
\end{array}\right], M=\left[\begin{array}{cc}
\frac{\omega_{h}}{\omega_{\alpha}} & 0 \\
0 & r_{\alpha}^{2}
\end{array}\right], \boldsymbol{f}=\left\{\begin{array}{c}
-c_{l} \\
2 c_{m}
\end{array}\right\}
$$

where $h, \alpha$ are the plunge and pitch displacements respectively, $x_{\alpha}$ is the dimensionless static unbalance, $r_{\alpha}$ is the dimensionless section moment of inertia, $\omega_{h}$ and $\omega_{\alpha}$ are the uncoupled natural frequencies in plunge and pitch respectively, and $c_{l}, c_{m}$ are the lift and moment coefficients at the elastic center. The elastic axis is placed at $e=0.2 c$. The velocity index $V_{F}$ is given here from $V_{F}=U_{\infty} /\left(\omega_{\alpha} b \sqrt{\mu}\right)$, where $b=c / 2$ is the semi-chord length, $\mu=m /\left(\pi \rho_{\infty} b^{2}\right)$ is the mass ratio and $U_{\infty}$ the freestream velocity.

Once again, the NACA 64A010 airfoil will be examined for a constant Mach number $M=0.8$ and a mean angle of attack $\alpha_{M}=0(\mathrm{deg})$. The airfoil structural parameters for this test case are shown in Table 2. The computational grid discussed in the previous test case has been employed also here. The flow calculations were carried out again with the Euler solver of SU2 and the same numerical parameters.

Table 2 Specifications for the Flutter/LCO prediction.

\begin{tabular}{cccc}
\hline Static Unbalance & Radius of gyration & Frequency ratio & Mass ratio \\
\hline$x_{\alpha}=0.25$ & $r_{\alpha}^{2}=0.75$ & $\omega_{h} / \omega_{\alpha}=0.5$ & $\mu=75$ \\
\hline
\end{tabular}

Multiple calculations were carried out in order to create the bifurcation diagram for the airfoil and predict the flutter onset. The pitch mode was chosen to be constrained in the search of the flutter frequency $\omega$ and reduced velocity $\tilde{V}=U_{\infty} / \omega_{\alpha} c$. Decreasing values of imposed pitch $\alpha_{\text {target }}$ were given as an input to the solver for the calculations. For each aeroelastic simulation, the displacement vector was initialized with the imposed pitch $\alpha_{\text {target }}$, and an initial plunge displacement $h_{0} / b=\alpha_{\text {target }} / \epsilon$, where $\epsilon<1$. The initial phase difference between the two motions was set at $\Delta \theta=5$ ( deg) for all the calculations, with the pitch mode always fixed at a zero phase. The initial frequency was chosen at $\omega_{0} / \omega_{\alpha}=0.7$, in between the natural frequencies of the system. The initial reduced velocity was set at $\tilde{V}_{0}=3.05$. The sensitivity of the prediction on the values $\tilde{V}_{0}$ and $\omega_{0}$ was evaluated and the procedure was found to be robust with respect to the initial conditions. At every aeroelastic computation, the inlet pressure is updated based on the $\tilde{V}$ prediction, for a fixed $M, \mu$ and $\rho_{\infty}$. 
Only one harmonic has been used for the solution of both the fluid and the structural problem. This is justified only close to the flutter onset where the displacement is small and the aerodynamics close to a linear state. Further away, the displacements become increasingly larger and more harmonics should be added. In the present study, however, the main focus was the prediction of the flutter onset. The use of only one harmonic significantly accelerated the procedure, and it was kept, thus, for all the calculations. It will be shown, however, that the accuracy of the computations was always satisfactory even when moving away from the flutter boundary. About $N_{C F D}=100-200$ iterations were carried out with the fluid solver in every aeroelastic iteration, while ten times more $N_{C F D}$ iterations were carried out for the initialization of the HB flow fields. The convergence of the complete procedure was achieved at about $N_{A E R O}=400$ aeroelastic iterations.

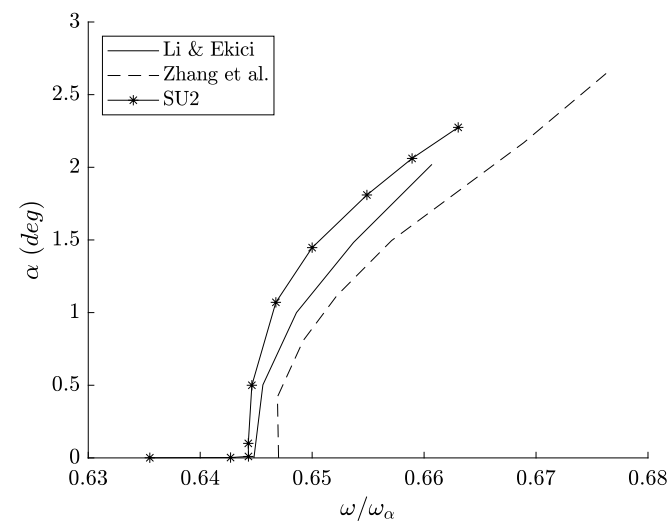

(a)

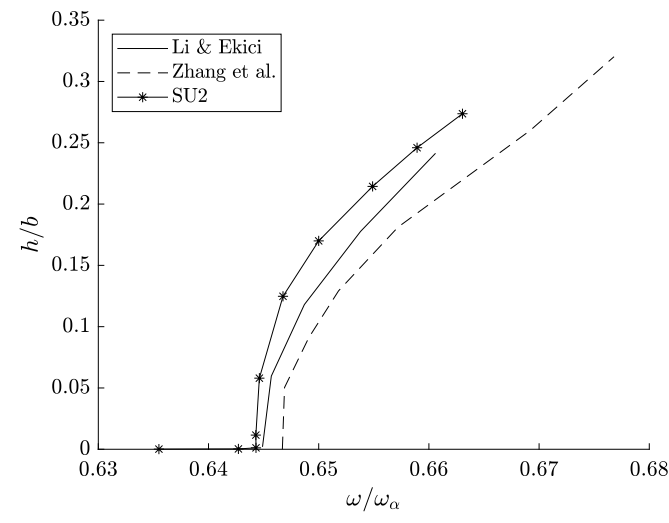

(c)

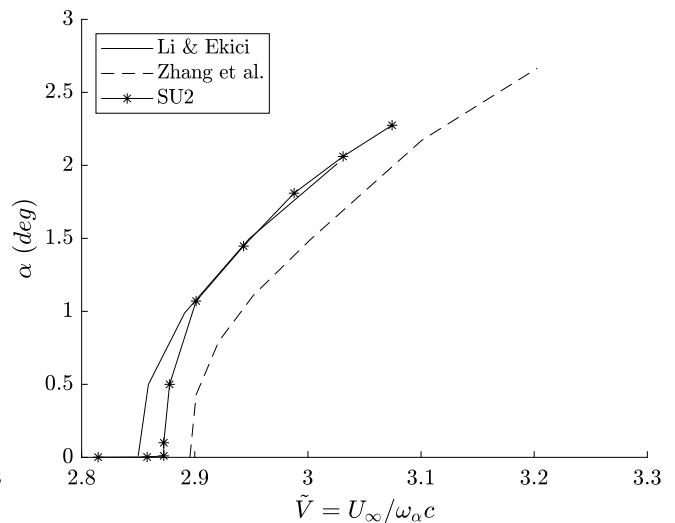

(b)

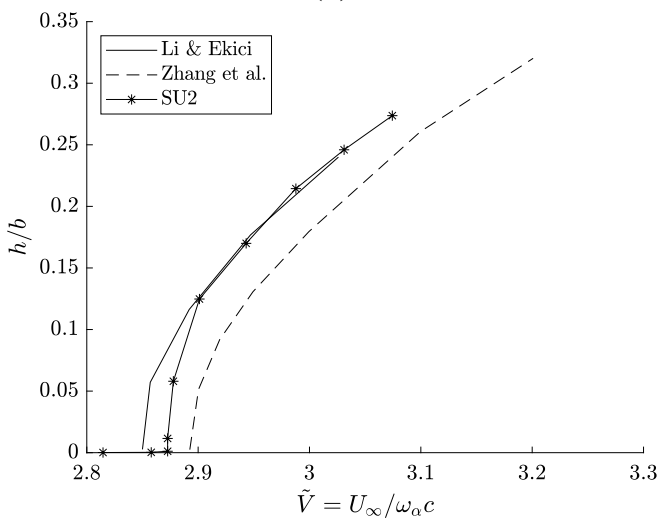

(d)

Fig. 3 Bifurcation diagram produced with SU2 compared with Refs. [10, 20], pitch (top) and plunge (bottom) amplitude against frequency (left), and reduced velocity (right).

The results from the flutter/LCO prediction with SU2 are presented in Fig 3, against available data in [10, 20]. Zhang et al. in [20] performed TM simulations, while Li \& Ekici [10] used their "one-shot" approach with one harmonic. The imposed pitching amplitude is shown in Fig 3a against the predicted frequency of the oscillation, while Fig $3 b$ shows the corresponding values of the reduced velocity. The calculated plunging amplitude is shown in Figs $3 \mathrm{c}$ and $3 \mathrm{~b}$ against the frequency and the reduced velocity, respectively. The typical parabolic shape of the bifurcation shape that was expected is well captured by the method. As the amplitude and the reduced velocity decrease, the system approaches the flutter boundary. Close to the flutter onset, the variation of the amplitude presents a linear response with respect to the reduced velocity, which is in agreement with the theory. Beyond the flutter point, and as the amplitude increases, the nonlinear LCO behavior appears. Both the frequency and the reduced velocity keep increasing with a decelerated rate. The results compare well with the references and clearly demonstrate the robustness and the accuracy of the solution procedure. The frequency seems to be underpredicted everywhere, with the difference, however, remaining below $1 \%$.

It was seen from this study that the solution procedure was quite sensitive to variations in the flow and the aerodynamic force prediction. Due to the apparition of shock waves over the airfoil in this transonic range, the flow 
convergence becomes slow. This affects significantly the convergence of the reduced velocity, and of the displacement amplitude, as the rate of the convergence follows exactly the reduction of flow residuals. In addition, the procedure was found to be numerically unstable when the flow residuals were not decreased enough at every aeroelastic iteration, relative to the previous one. As a result, the choice of numerical schemes should be done carefully considering the flow characteristics. Finally, this discussion shows that if better suited CFD grids were to be used (e.g., adapted to shocks), it could be possible to decrease the $N_{C F D}$ iterations even further and accelerate the $\tilde{V}$ and $\omega$ convergence.

\section{Flutter boundary of the AGARD 445.6 wing}

In this section, the weakened AGARD 445.6 model $3[21]$ is considered. The wing uses the symmetric NACA 64A004 profile, with a maximum thickness of 4\%, and has a sweep angle of $45(\mathrm{deg})$ at the quarter chord line. The span of the wing is $s=0.726 m$ with a taper ratio $T R=0.66$, where the root chord is $c_{r}=2 b_{r}=0.5588$. A rectangular unstructured computational grid is build around the wing geometry which is clamped on one of the $x z$ sides of the domain. The dimensions of the computational domain are $D_{x} \times D_{y} \times D_{z}=14 c_{r} \times 6 c_{r} \times 12 c_{r}$, where $x$ is positive in the stream-wise direction, and the $y$-wise direction is placed along the span of the wing. The aerodynamic surface mesh of the model consists of 65000 boundary elements and is shown in Fig $4 \mathrm{a}$. The total grid size was about 320000 volume elements.

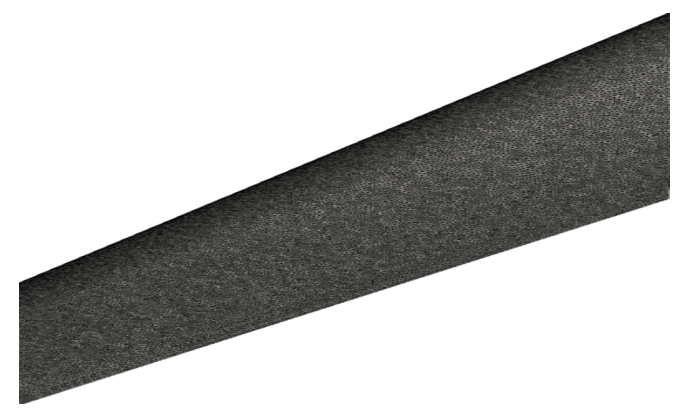

(a)

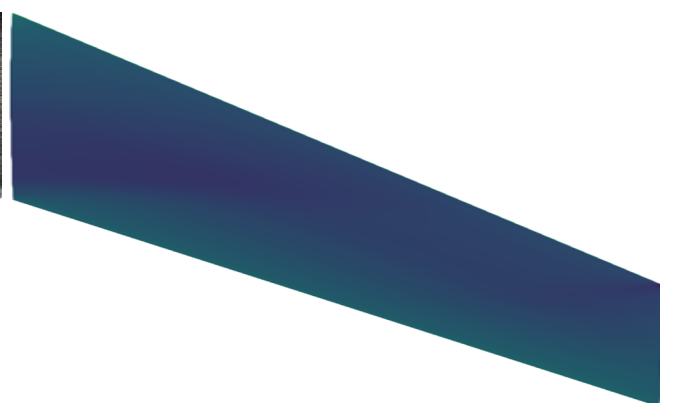

(b)

Fig. 4 AGARD 445.6 wing, (a) the aerodynamic surface mesh, and (b) the pressure distribution on the surface of the wing for $M=0.96$ at $a_{0}=0$.

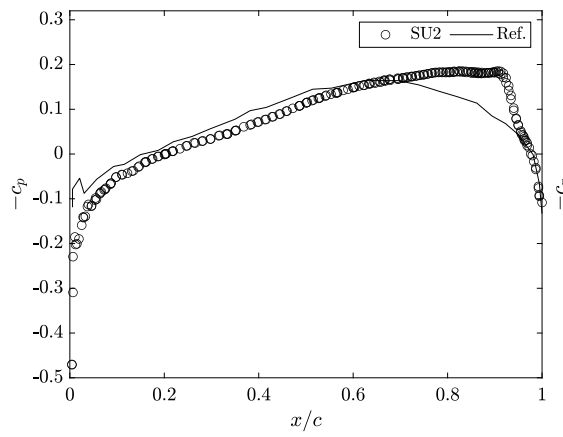

(a)

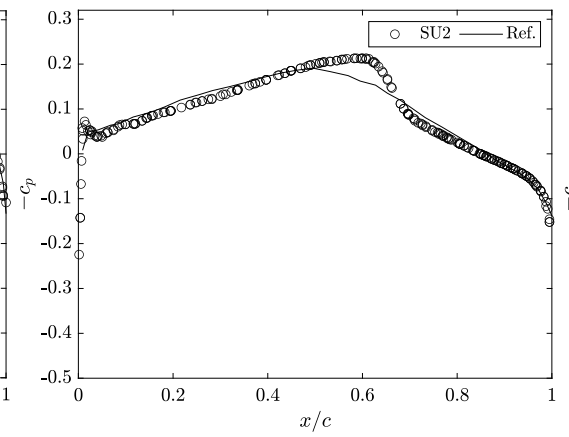

(b)

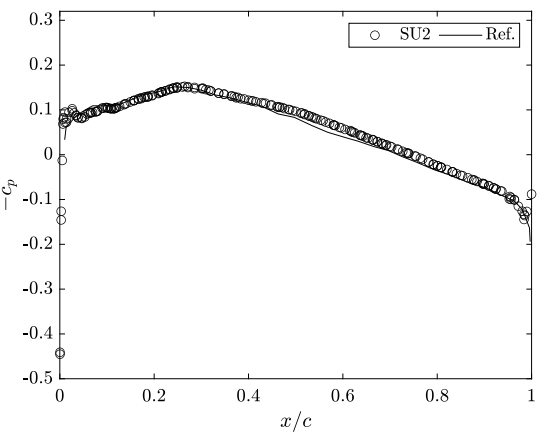

(c)

Fig. 5 Pressure coefficient $c_{p}$ at three span-wise stages: (a) $y / s=0$, (b) $y / s=0.25$, and (c) $y / s=0.9$, steady-state results compared with Ref. [22].

The aerodynamic model is first evaluated at $M=0.96, \rho_{\infty}=0.063 \mathrm{~kg} / \mathrm{m}^{3}$ and at a zero angle of attack. Steady Euler calculations are carried out with freestream boundary conditions at the sides of the domain, and slip wall conditions on the wing. The JST central scheme with second and fourth order dissipation coefficients was employed for this study as well. The simulation converges fast and the lift coefficient is driven to the expected zero value after only $\approx 150$ iterations. The computed pressure distribution is shown on the surface of the wing in Fig. 4b. The shock front along the span is highlighted by the pressure jumps on the surface of the wing. The surface pressure coefficient $c_{p}$ is plotted for three 
different positions along the span, and is shown in Fig. 5] The results are compared with the ones found in [22]. It can be seen that for stages close to the root of the wing, SU2 predicts stronger and sharper shocks compared to Ref. [22]. This is probably due to the fine surface mesh used in the current study, resulting in low levels of numerical dissipation. In later span-wise stages were the shock is smeared, the prediction is in great agreement with the literature. Finally, two even finer meshes have been created and investigated, providing equivalent results.

The modal description of the structural model is taken from [23]. The modal shapes are given on a $11 \times 11$ plane grid, and were normalised to a unit modal mass in $1 \mathrm{bf}-\mathrm{in}-\mathrm{s}^{2}$, or $175.13 \mathrm{~kg}$. The first four normal modes are used in this study. The respective natural frequencies are $f=\left[f_{1}, f_{2}, f_{3}, f_{4}\right]=[9.6,38.17,48.35,91.54] \mathrm{Hz}$. Using the RBF implementation, described in Section III.D with the Euclid's hat basis function, the modal shapes are interpolated onto the aerodynamic surface mesh of the wing. The first two modes on the aerodynamic surface are shown in Fig. 6, with respect to the undeformed mean surface. The interpolated displacement follows the imposed structural deformation with remarkable accuracy.

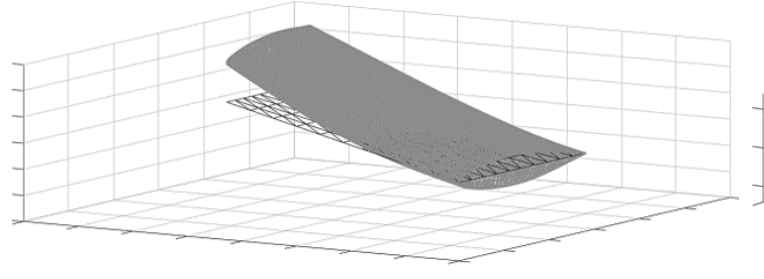

(a)

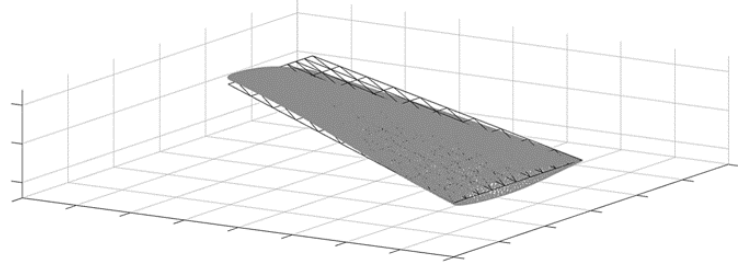

(b)

Fig. 6 The first (a) bending, and (b) torsion mode shapes interpolated onto the aerodynamic surface mesh.

Next, the native implementation of the reduced order structural solver in SU2 is validated. The static aeroelastic equilibrium is searched for $M=0.8, \rho_{\infty}=0.0941 \mathrm{~kg} / \mathrm{m}^{3}$ and an $a_{1}=1(\mathrm{deg})$ angle of attack. A second-order dual time-stepping is performed for the convergence to the steady-state problem. A relatively large time-step $\Delta t=0.1 \mathrm{~s}$ is used since the problem is expected to converge to a static equilibrium. At every time step, 50 inner iterations are performed for the flow equations. At the end of every outer time-step, the dynamic aeroelastic equations are solved. The rest of the numerical parameters are as described before. The problem converges to the static equilibrium at about 20 - 30 time-steps. The wing-tip displacement is shown in Fig. 7] against the results found in [24]. The agreement with the literature is quite satisfactory. Only small differences appear and could be attributed to the interpolation technique and/or the CFD mesh.

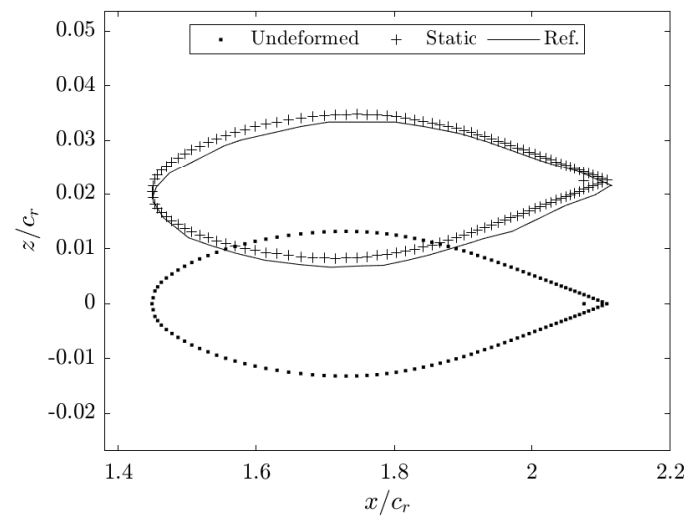

Fig. 7 Wing-tip displacement at the static equilibrium for $M=0.8$ and $a_{1}=1$ (deg), results compared with Ref. [24].

Finally, the flutter boundary of the wing is predicted here. Six different Mach numbers are investigated and are shown in Table 3 . For each case, the freestream density $\rho_{\infty}$ is fixed and the mass ratio $\mu$ is calculated accordingly in order to keep the mass of the wing panel constant. The freestream pressure at the flutter onset is searched for through 
the updates of the flutter velocity. The first bending mode $q_{1}$ was chosen to be constrained here. A sufficiently small value $\left\|q_{1}\right\|_{\text {target }}=0.001$ was given as an input to the solver for all the calculations. The phase of $q_{1}$ was always fixed at a zero phase. For each aeroelastic simulation, the displacement vector was initialized with the imposed $\left\|q_{1} \mid\right\|_{\text {target }}$, and zero generalized displacements for the rest of the modes. For all the calculations, the initial frequency was chosen at $\omega_{0} / \omega_{2}=0.34$, were $\omega_{2}$ is the natural frequency of the second mode, i.e. the first torsion mode. The initial reduced velocity was set at $\left(V_{F}\right)_{0}=0.5$. The sensitivity of the prediction on the values $V_{F}$ and $\omega$ was evaluated. It was found that the procedure remained quite insensitive to changes of the initial $V_{F}$, but was unstable when $\omega_{0} \leq \omega_{1}$, and also when $\omega_{0}$ values approached $\omega_{2}$.

Table 3 Specifications for the Flutter/LCO prediction.

\begin{tabular}{ccccccc}
\hline & 1 & 2 & 3 & 4 & 5 & 6 \\
\hline$M$ & 0.499 & 0.678 & 0.799 & 0.901 & 0.960 & 1.072 \\
$\rho_{\infty}$ & 0.4267 & 0.2077 & 0.1535 & 0.0992 & 0.0633 & 0.055 \\
$\mu$ & 33.456 & 68.753 & 93.024 & 143.92 & 225.82 & 259.59 \\
\hline
\end{tabular}

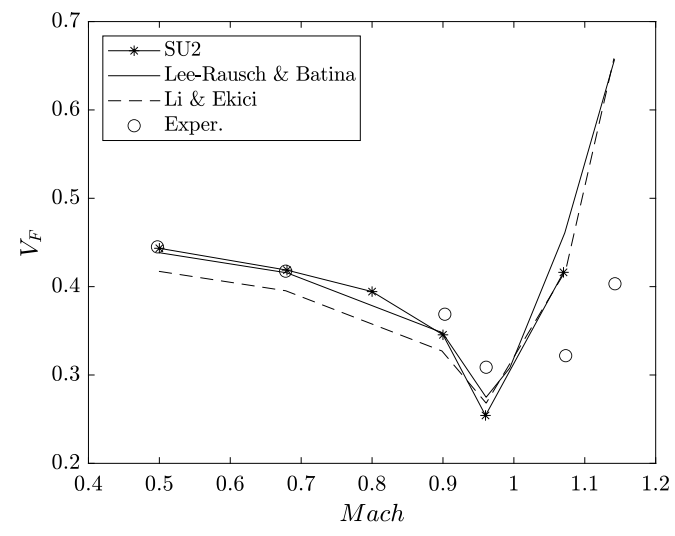

(a)

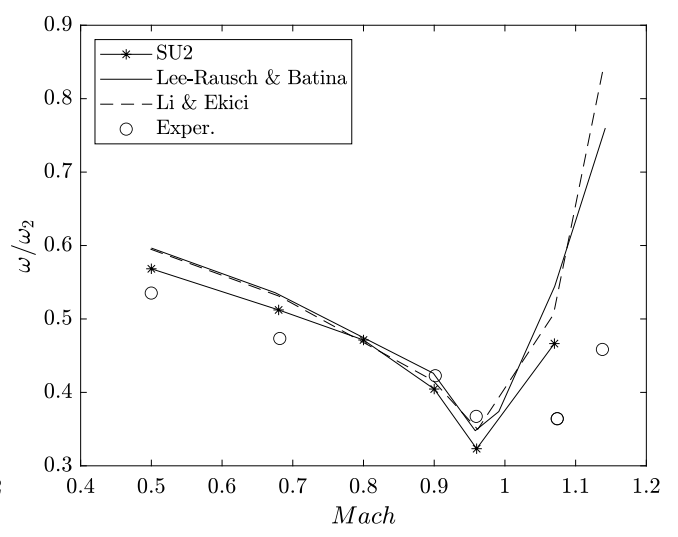

(b)

Fig. 8 Flutter boundary of the AGARD 445.6 model 3, prediction of the (a) flutter velocity index, and (b) the nondimensional frequency, comparison with Refs. [23, 25, 26]

Only one harmonic has been used for both the fluid and the structural problem, which is justified close to the flutter onset where the displacement is small. About $N_{C F D}=100$ iterations were carried out with the fluid solver in every aeroelastic iteration. Four times more iterations were carried out for the initialization of the HB flow fields. The convergence of the complete procedure was achieved at about $N_{A E R O}=800$ aeroelastic iterations for $0.8<M<1.0$. Outside this range, either shocks do not form, or they form at the aft part of the wing profile sections. This significantly accelerates the convergence of the flow solution and, thus, the whole flutter prediction converges at about 100 aeroelastic iterations. As mentioned in the previous section, the convergence of $V_{F}$ and $\omega$ prediction strongly relates to the decrease of flow residuals.

The prediction of the flutter boundary is shown in Fig. 8. For variable Mach values, the flutter velocity index $V_{F}$ is shown in Fig. 8a. while the nondimensional frequency $\omega / \omega_{2}$ is shown in Fig. 8b. The results are plotted against Euler computations from [25], the "one-shot" approach of [26], and the experimental data found in [23]. The transonic deep at $M=0.96$ is well captured with the SU2 implementation. Overall, the agreement with the literature is satisfactory. The flutter velocity index follows exactly the prediction in [25] until the transonic deep. A slight underestimation of the index is seen at $M=0.96$. The method slightly underpredicts the frequency compared to both [25] and [26] for every $M<1$, but not compared to the experimental results found in [23]. Finally, all the numerical cases reduce in accuracy compared to the experimental results for $M>1$. This should be attributed to the complex nonlinear aerodynamics arising from the shock-wave boundary layer interactions, and the shortcomings of the specific CFD modelling. 


\section{Conclusions}

The state-of-the-art on frequency-domain techniques for CFD-based flutter prediction has been briefly discussed in this paper. The motivation behind the development of such techniques comes from the robustness and the efficiency that they offer when searching directly for the flutter boundary across the flight envelope. Based on the literature review, a flutter prediction framework has been developed in the open-source SU2 suite and demonstrated here for standardized $2 \mathrm{D}$ and $3 \mathrm{D}$ geometries.

The complete framework employs dedicated interpolation schemes to transfer data across the fluid-structure interface. The implementation includes a native reduced order structural solver that has been validated against the existing literature. In addition, the existing HB formulation in SU2 has been successfully extended to treat arbitrarily deforming surfaces and volume meshes. For both the 2D and 3D cases investigated in this work, the flutter prediction strategy was found to be accurate and relatively insensitive to the change of initial conditions.

The robustness of the current implementation permits the modification of the current approach in order to account for structural nonlinearities. These may appear at flight conditions where the mean displacement becomes important, altering the modal shapes of the wing. Future research will examine ways to include the current implementation within an optimization framework, where the flutter sensitivity of the configuration poses additional constraints onto the design process. To this end, the SU2 suite, which is an efficient multi-physical design tool, offers an ideal environment.

\section{References}

[1] Cesnik, C. E. S., Palacios, R., and Reichenbach, E. Y., "Re-examined Structural Design Procedures for Very Flexible Aircraft," Journal of Aircraft, Vol. 51, No. 5, 2014, pp. 1580-1591. https://doi.org/10.2514/1.C032464

[2] Simiriotis, N., Fragiadakis, M., Rouchon, J.-F., and Braza, M., "Shape control and design of aeronautical configurations using shape memory alloy actuators," Computers \& Structures, Vol. 244, 2021, p. 106434. https://doi.org/https://doi.org/10.1016/j. compstruc.2020.106434.

[3] Hall, K. C., Thomas, J. P., and Clark, W. S., "Computation of Unsteady Nonlinear Flows in Cascades Using a Harmonic Balance Technique," AIAA Journal, Vol. 40, No. 5, 2002, pp. 879-886. https://doi.org/10.2514/2.1754

[4] McMullen, M., and Jameson, A., "The computational efficiency of non-linear frequency domain methods," Journal of Computational Physics, Vol. 212, No. 2, 2006, pp. 637-661. https://doi.org/10.1016/j.jcp.2005.07.021

[5] Gopinath, A., and Jameson, A., "Time Spectral Method for Periodic Unsteady Computations over Two- and Three- Dimensional Bodies,", 2005. https://doi.org/10.2514/6.2005-1220

[6] Thomas, J. P., Dowell, E. H., and Hall, K. C., "Nonlinear Inviscid Aerodynamic Effects on Transonic Divergence, Flutter, and Limit-Cycle Oscillations," AIAA Journal, Vol. 40, No. 4, 2002, pp. 638-646. https://doi.org/10.2514/2.1720

[7] Thomas, J. P., and Dowell, E. H., "A Fixed Point Iteration Approach for Harmonic Balance Based Aeroelastic Computations," 2018 AIAA/ASCE/AHS/ASC Structures, Structural Dynamics, and Materials Conference, 2018. https://doi.org/10.2514/6.2018-1446

[8] He, S., Jonsson, E., Mader, C. A., and Martins, J. R. R. A., “A Coupled Newton-Krylov Time Spectral Solver for Wing Flutter and LCO Prediction," AIAA 2019-3549, AIAA Aviation 2019 Forum, 2018. https://doi.org/10.2514/6.2019-3549

[9] Ekici, K., and Hall, K. C., "Harmonic Balance Analysis of Limit Cycle Oscillations in Turbomachinery," AIAA Journal, Vol. 49, No. 7, 2011, pp. 1478-1487. https://doi.org/10.2514/1.J050858.

[10] Li, H., and Ekici, K., "Revisiting the One-shot method for modeling limit cycle oscillations: Extension to two-degree-of-freedom systems," Aerospace Science and Technology, Vol. 69, 2017, pp. 686-699. https://doi.org/10.1016/j.ast.2017.07.037

[11] Li, H., and Ekici, K., "Improved One-Shot Approach for Modeling Viscous Transonic Limit Cycle Oscillations," AIAA Journal, Vol. 56, No. 8, 2018, pp. 3138-3152. https://doi.org/10.2514/1.J056969.

[12] Li, H., and Ekici, K., "A novel approach for flutter prediction of pitch-plunge airfoils using an efficient one-shot method," Journal of Fluids and Structures, Vol. 82, 2018, pp. 651-671. https://doi.org/10.1016/j.jfluidstructs.2018.08.012

[13] Economon, T. D., Palacios, F., Copeland, S. R., Lukaczyk, T. W., and Alonso, J. J., "SU2: An Open-Source Suite for Multiphysics Simulation and Design,” AIAA Journal, Vol. 54, No. 3, 2016, pp. 828-846. https://doi.org/10.2514/1.J053813.

[14] Rubino, A., Pini, M., Colonna, P., Albring, T., Nimmagadda, S., Economon, T., and Alonso, J., “Adjoint-based fluid dynamic design optimization in quasi-periodic unsteady flow problems using a harmonic balance method," Journal of Computational Physics, Vol. 372, 2018, pp. 220-235. https://doi.org/10.1016/j.jcp.2018.06.023 
[15] de Boer, A., van Zuijlen, A., and Bijl, H., "Review of coupling methods for non-matching meshes," Computer Methods in Applied Mechanics and Engineering, Vol. 196, No. 8, 2007, pp. 1515-1525. https://doi.org/https://doi.org/10.1016/j.cma.2006.03.017. domain Decomposition Methods: recent advances and new challenges in engineering.

[16] Beckert, A., and Wendland, H., "Multivariate interpolation for fluid-structure-interaction problems using radial basis functions," Aerospace Science and Technology, Vol. 5, No. 2, 2001, pp. 125-134. https://doi.org/https://doi.org/10.1016/S12709638(00)01087-7

[17] Rendall, T. C. S., and Allen, C. B., "Unified fluid-structure interpolation and mesh motion using radial basis functions," International Journal for Numerical Methods in Engineering, Vol. 74, No. 10, 2008, pp. 1519-1559. https://doi.org/https: //doi.org/10.1002/nme.2219

[18] Thomas, D., Cerquaglia, M., Boman, R., Economon, T., Alonso, J., Dimitriadis, G., and Terrapon, V., "CUPyDO - An integrated Python environment for coupled fluid-structure simulations," Advances in Engineering Software, Vol. 128, 2019 , pp. 69-85. https://doi.org/https://doi.org/10.1016/j.advengsoft.2018.05.007

[19] McMullen, M., Jameson, A., and Alonso, J., "Demonstration of Nonlinear Frequency Domain Methods," AIAA Journal, Vol. 44, No. 7, 2006, pp. 1428-1435. https://doi.org/10.2514/1.15127

[20] Zhang, W., Wang, B., Ye, Z., and Quan, J., "Efficient Method for Limit Cycle Flutter Analysis Based on Nonlinear Aerodynamic Reduced-Order Models,” AIAA Journal, Vol. 50, No. 5, 2012, pp. 1019-1028. https://doi.org/10.2514/1.J050581

[21] Yates, E. C., "AGARD standard aeroelastic configurations for dynamic response. Candidate configuration I.-wing 445.6," Proceedings of the 61st meeting of the Structures and Materials Pannel, 1987, pp. 1-73.

[22] Beaubien, R., Nitzsche, F., and Feszty, D., "Time and frequency domain flutter solutions for the AGARD 445.6 wing," Paper IF-102, IFASD, 2005.

[23] Yates, E. C., Land, N. S., and Foughner Jr., J. T., "Measured and calculated subsonic and transonic flutter charactersitscs of a 45 deg. sweptback wing planform in air and in Freon-12 in the Langley Transonic Dynamics Tunnel," NASA Langley Reasearch Center, Tech. Rep., 1963, pp. NASA Technical Note D-1616.

[24] Melville, R., Morton, S., Rizzetta, D., Melville, R., Morton, S., and Rizzetta, D., "Implementation of a fully-implicit, aeroelastic Navier-Stokes solver," AIAA, 13th Computational Fluid Dynamics Conference, 1997.

[25] Lee-Rausch, E. M., and Batina, J. T., "Wing flutter boundary prediction using unsteady Euler aerodynamic method," Journal of Aircraft, Vol. 32, No. 2, 1995, pp. 416-422. https://doi.org/10.2514/3.46732

[26] Li, H., and Ekici, K., "Aeroelastic Modeling of the AGARD 445.6 Wing Using the Harmonic-Balance-Based One-Shot Method," AIAA Journal, Vol. 57, No. 11, 2019, pp. 4885-4902. https://doi.org/10.2514/1.J058363. 